\title{
Clinicopathological and patient characteristics of early gastric neoplasia endoscopically resected with loss of Mlh1 expression
}

\author{
SHUJI SASAKI ${ }^{1}$, KAZUO YASHIMA $^{1}$, AKIHIRO HAYASHI ${ }^{1}$, YOHEI TAKEDA ${ }^{1}$, \\ AKIKO YASUGI $^{1}$, MASAHARU KODA ${ }^{1}$, KOICHIRO KAWAGUCHI ${ }^{1}$, \\ KENICHI HARADA $^{1}$, HISAO ITO ${ }^{2}$ and YOSHIKAZU MURAWAKI ${ }^{1}$ \\ Divisions of ${ }^{1}$ Medicine and Clinical Science and ${ }^{2}$ Organ Pathology, \\ Faculty of Medicine, Tottori University, Yonago 683-8504, Japan
}

Received September 15, 2010; Accepted December 6, 2010

DOI: $10.3892 / \mathrm{ol} .2010 .231$

\begin{abstract}
Hypermethylation of the promoter region of the MLH1 gene leads to loss of Mlh1 protein expression and plays a key role in the development of gastric cancer. Little is known about the association between Mlh1 expression and the clinicopathological and patient characteristics in early gastric neoplasia, particularly in endoscopically resected tumors. Immunohistochemistry was used to examine Mlh1 expression in 140 early gastric neoplasias obtained by endoscopic resection and comprising 31 gastric adenomas (GAs) and 109 early gastric cancers (EGCs), and compared them to corresponding clinicopathological and patient data. P53 expression and phenotypic profiles were also analyzed. The rate of reduced Mlh1 expression and P53 overexpression was 9.6 and $6.5 \%$ in GAs, and 27.5 and $27.5 \%$ in EGCs, respectively. In elderly patients ( $\geq 65$ years of age), the aberrant expression of Mlh1 in EGCs was more significant in female than in male patients (59.9 vs. $29.8 \%$; $P=0.016$ ). In addition, the frequency of aberrant Mlh1 expression in EGCs increased significantly in patients with oncological family histories and elevated gross type ( $\mathrm{P}=0.033$ and $\mathrm{P}=0.04$, respectively). Moreover, a significant correlation was observed among aberrant Mlh1, P53-negative and HGM expression. The present findings suggest that loss of Mlh1 expression is associated with age, gender, oncological family history and tumor growth pattern in EGC. Patient and tumor characteristics are key factors in the screening, surveillance and diagnosis of early gastric neoplasia, particularly in elderly individuals.
\end{abstract}

\section{Introduction}

The incidence of gastric cancer (GC) has decreased over the last decade. However, GC remains the second leading cause

Correspondence to: Dr Kazuo Yashima, Division of Medicine and Clinical Science, Faculty of Medicine, Tottori University, 36-1 Nishicho, Yonago 683-8504, Japan

E-mail: yashima@grape.med.tottori-u.ac.jp

Key words: gastric cancer, Mlh1, P53, endoscopic resection of cancer-related mortality and the fourth most common malignant tumor type worldwide $(1,2)$. The incidence of GC in elderly individuals is currently on the increase in Japan due to the extended life span of the general population. It is estimated that GC in patients 65 years of age or older accounts for approximately $70 \%$ of total GCs (3). GC risk is affected by $H$. pylori virulence factors, a family history of GC, host genetics and environmental factors (cigarette smoking, in particular) (4-7). A high mortality rate has been noted worldwide, with a 5-year survival rate of approximately $20 \%$ (8). In Japan, the 5-year survival rate is over 50\% (9). One of the key factors that negatively affect the survival rate is the late detection of tumors. To achieve a complete cure, primary tumors should be detected at an early stage. It is therefore crucial to acquire a better understanding of the clinicopathological and patient characteristics in early gastric neoplasia.

GC develops through the accumulation of genetic and epigenetic alterations (10). In sporadic GC, the frequency of DNA methylation of $M L H 1$ is $20-30 \%$ (11-13), and that of the $P 53$ gene mutation is $25-50 \%(12,14,15)$. MLH1 is a DNA mismatch repair (MMR) gene and hypermethylation of the promoter region of $\mathrm{MLHI}$ is the main cause of microsatellite instability (MSI) in primary GCs (16). Fleisher et al reported that immunohistochemical staining of the Mlh1 protein may be included in routine diagnostic methods used to predict hypermethylation of the $M L H 1$ promoter (17). This suggests that the simple detection of MSI caused by the DNA methylation of MLH1 is achieved by immunohistochemical analysis of tumor specimens. A number of clinicopathological characteristics of GC with MMR-gene deficiency or MSI were previously reported (18-21). However, the relationship between MMR abnormality and the clinicopathological characteristics in early-stage gastric neoplasia, particularly in endoscopically resected samples, has yet to be elucidated.

Mucin-based histochemical and immunohistochemical examinations showed that gastric and intestinal phenotypic cell markers are widely expressed in GCs, irrespective of histological type (22-24). Moreover, it was reported that GCs with a predominantly gastric phenotype have a pronounced tendency toward invasion, metastasis and poor prognosis compared to GCs that have an intestinal phenotypic expression (25-28). 
Elucidation of the relationship between the clinicopathological and patient characteristics and the molecular events in early gastric neoplasia may improve the early detection, treatment and surveillance of GC. The relationship between Mlh1 expression and the clinicopathological characteristics, such as age, gender, alcohol consumption, smoking, oncological family history, P53 expression and phenotypic expression in early stages of gastric neoplasias, were evaluated using tumor tissues removed by endoscopic resection (ER).

\section{Materials and methods}

Patients and tissue samples. Tumor specimens were obtained from 140 patients (89 males and 51 females) who underwent ER at Tottori University Hospital, Japan, between 1994 and 2007. Patients with familial adenomatous polyposis or hereditary non-polyposis colorectal cancer were excluded. The histological examination showed that of the 140 tumors, 31 were gastric adenomas (GAs) and 109 early gastric cancers (EGCs) (Table I). Macroscopic and histological evaluations were performed according to the classifications of the Japanese Gastric Cancer Association (29). Macroscopic features were divided into two major types: elevated and flat or depressed. The depth of invasion and histological grade were classified according to the predominant features of the tumors. In this study, the adenoma samples corresponded to moderate or high-grade adenoma/dysplasia and the cancer samples corresponded to non-invasive or intramucosal carcinoma according to the Vienna classification system (30), with the exception of seven submucosal invasive cancers. The EGCs comprised histologically differentiated carcinomas, with the exception of four poorly differentiated and/or signet-ring cell carcinomas. H. pylori positivity was not checked. Two experienced pathologists (K.Y. and H.I.) verified the pathological diagnoses. Smoking habits, alcohol consumption and oncological family history of the patients were recorded by medical doctors on admission. Alcohol consumption and smoking were defined as regular intake when the respective consumption was $>35 \mathrm{~g}$ of ethanol and 5 cigarettes per day. Positivity for family history of cancer was defined as a history of cancer within first-degree relatives. Patients with a family history of gastric cancer and those with an oncological family history were identified. The cases were analyzed anonymously; specimens were assigned a number without any personal information. Approval for the study was obtained from the Institutional Review Board.

Immunohistochemical staining. Paraffin-embedded, 4- $\mu \mathrm{m}$ sections were immunohistochemically stained with anti-Mlh1 mouse monoclonal antibody (G168-15; PharMingen, San Diego, CA, USA; dilution 1:50), anti-P53 mouse monoclonal antibody (DO-7; Dakopatts, Copenhagen, Denmark; dilution 1:50), anti-human gastric mucin (HGM) mouse monoclonal antibody (45M1; Novocastra Laboratories, Ltd., Newcastle, UK; dilution 1:50), anti-MUC2 mouse monoclonal antibody (Ccp58; Novocastra; dilution 1:100) and anti-CD10 mouse monoclonal antibody (56C6; Novocastra; dilution 1:50) using the avidinbiotin-peroxidase complex technique.

Immunohistochemical staining was performed as described below. In brief, following deparaffinization in xylene and rehydration in ethanol, the sections were immersed in citrate
Table I. Clinicopathological characteristics and patient background in early gastric neoplasias.

\begin{tabular}{|c|c|c|}
\hline & $\begin{array}{c}\text { Early gastric } \\
\text { carcinoma }(n=109)\end{array}$ & $\begin{array}{l}\text { Gastric adenoma } \\
\qquad(\mathrm{n}=31)\end{array}$ \\
\hline Gender (M:F) & $75: 34$ & $14: 17$ \\
\hline Age $($ mean $\pm \mathrm{SD})$ & $69.7 \pm 9.1$ & $71.1 \pm 6.4$ \\
\hline Elevated/flat, depressed & $59: 50$ & $31: 0$ \\
\hline \multicolumn{3}{|l|}{ Histological type } \\
\hline & Tub 84 & Mild $\quad 1$ \\
\hline & Pap 21 & Moderate 20 \\
\hline & Por/Sig 4 & Severe $\quad 10$ \\
\hline $\begin{array}{l}\text { Depth of invasion } \\
\text { (mucosa:submucosa) }\end{array}$ & $102: 7$ & \\
\hline $\begin{array}{l}\text { Regular alcohol } \\
\text { intake }(\%)\end{array}$ & $51(46.8)$ & $8(35.8)$ \\
\hline $\begin{array}{l}\text { Current or ever } \\
\text { smoker }(\%)\end{array}$ & $58(53.2)$ & $13(41.9)$ \\
\hline $\begin{array}{l}\text { Oncological family } \\
\text { history }(\%)\end{array}$ & $61(60.0)$ & $20(64.5)$ \\
\hline $\begin{array}{l}\text { Family history of } \\
\text { gastric cancer }(\%)\end{array}$ & $42(38.5)$ & $12(38.7)$ \\
\hline
\end{tabular}

SD, stadard deviation; Tub, tubular adenocarcinoma; Pap, papillary adenocarcinoma; Por, poorly differentiated adenocarcinoma; Sig, signet ring cell adenocarcinoma.

buffer (0.01 M, pH 6.0) and heated in a microwave oven for 20-30 min to retrieve antigens. Endogenous peroxidase activity was blocked by incubation with $3 \% \mathrm{H}_{2} \mathrm{O}_{2}$ and the sections were then incubated with the primary antibody overnight at $4^{\circ} \mathrm{C}$. As a negative control, the primary antibody was replaced with normal serum $\operatorname{IgG}$ at a similar dilution. The detection reaction followed the protocol of Vectastain Elite ABC kit (Vector Laboratories, Burlingame, CA, USA). Diaminobenzidine was used as a chromogen and haematoxylin was used as a counterstain. The sections were incubated with biotinylated anti-mouse IgG and avidin-biotin-peroxidase, and then visualized using diaminobenzidine tetrahydrochloride.

Two independent observers (S.S. and K.Y.) evaluated the expression of the proteins tested and the immunohistochemical analysis was performed in a blind manner with respect to the clinical information.

Assessment of Mlh1 immunostaining. Evaluation of Mlh1 expression was regarded as either normal or decreased. The cases with definite nuclear staining in $<30 \%$ of the tumor cells were identified as decreased (Fig. 1).

Assessment of P53 immunostaining. Five representative fields were examined and a total of 1,000 tumor cells (200 for each field) were counted under the microscope using a highpower (x200) objective. Distinct nuclear immunoreaction was assessed as positive. In this study, the specimens were regarded as P53-positive when $>10 \%$ of tumor cells stained as positive. 
A

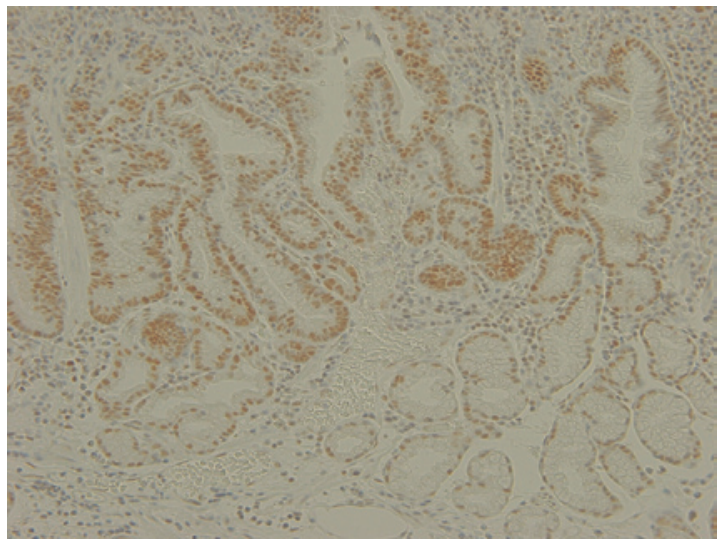

B

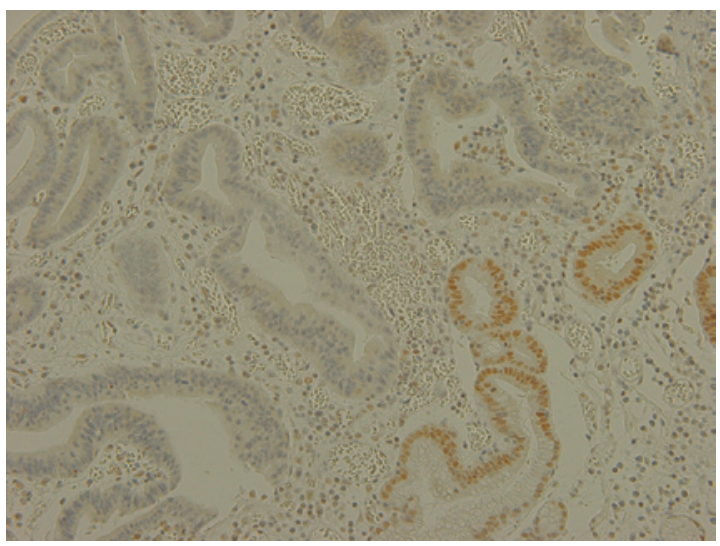

Figure 1. Expression of Mlh1 in early gastric cancer (GC). Mlh1 protein expression was examined immunohistochemically. (A) Sample from a 61-year-old male presenting with early gastric cancer with strong nuclear immunoreactivity for Mlh1 protein. (B) Sample from a 76-year-old female presenting with early gastric cancer with no nuclear immunoreactivity for Mlh1 protein. Normal mucosa with strong nuclear expression of Mlh1 protein is presented on the right side of each image.

Assessment of HGM, MUC2 and CD10 immunostaining. HGM staining was observed in the cytoplasm of the gastric foveolar epithelium and mucous neck cells. MUC2 staining was observed in the cytoplasm around the nuclei of the goblet cells and CD10 staining was observed along the brush border of the luminal surface of the epithelium. Although CD10 is also expressed in the apical portion of the cytoplasm of normal gastric mucosa, only the expression of CD10 along the brush border was studied. The results from the staining were classified as positive and negative expression. Staining of $>10 \%$ of the adenoma and carcinoma cells was regarded as having a positive expression and $<10 \%$ as having a negative expression.

Statistical analysis. Statistical analysis was performed using the Chi-square test with Yates' correction, Fisher's test, the Mann-Whitney $\mathrm{U}$ test and logistic regression. $\mathrm{P}<0.05$ was considered to be statistically significant. Statistical computations were performed using the Stat View 5.0 software (SAS Institute, Cary, NC, USA).

\section{Results}

Clinicopathological and patient characteristics. Clinicopathological characteristics and patient backgrounds in early gastric neoplasias are shown in Table I. The mean age of the
Table II. Mlh1, P53 and phenotype expression in early gastric neoplasias.

\begin{tabular}{lccc}
\hline & Cancer $(\mathrm{n}=109)$ & \multicolumn{2}{c}{ Adenoma $(\mathrm{n}=31)$} \\
\hline Mlh1 (+/-) & $79: 30(27.5 \%)$ & $28: 3$ & $(9.6 \%)$ \\
P53 (+/-) & $30: 79(27.5 \%)$ & $2: 29(6.5 \%)$ \\
HGM (+/-) & $71: 38(65.1 \%)$ & $10: 21(32.3 \%)$ \\
CD10 (+/-) & $30: 79(27.5 \%)$ & $21: 10(66.7 \%)$ \\
MUC2 (+/-) & $53: 56(48.6 \%)$ & $27: 4 \quad(87.1 \%)$ \\
\hline
\end{tabular}

EGC cases was $69.7 \pm 9.1$ years (range 45-85) and that of the GA cases was 71.1 \pm 6.4 years (range $51-82$ ). GAs were found more often in female than in male patients (33.3 vs. $15.7 \%$; $\mathrm{P}=0.016)$. Among the 140 gastric neoplasia cases, 54 (38.5\%) had a family history of GC and $81(57.9 \%)$ had a family history of malignancy. A total of 59 patients $(42.1 \%)$ consumed alcohol and 71 patients (50.7\%) were smokers.

Mlh1, P53 and phenotypic (HGM, MUC2, CD10) expression in early gastric neoplasias. Loss of Mlh1 expression was found more often in EGCs than in GAs [30/109 (27.5\%) vs. $3 / 31$ (9.6\%), $\mathrm{P}=0.053]$. P53 overexpression occurred significantly more often in EGCs than in GAs [30/109 (27.5\%) vs. 2/31 (6.5\%), P=0.014]. Significant HGM expression was found more often in EGCs than in GAs [71/109 (65.1\%) vs. 10/31 (32.3\%), P=0.001]. On the other hand, CD10 and MUC2 expression occurred significantly more often in GAs than in EGCs [21/31 (66.7\%) vs. 30/109 (27.5\%); $\mathrm{P}<0.001$ and 27/31 (87.1\%) vs. 53/109 (27.5\%); $\mathrm{P}=0.001$, respectively] (Table II).

Relationship between Mlh1 expression and the clinicopathological and patient characteristics in early gastric neoplasias. Female patients presented more often with EGCs showing a loss of Mlh1 expression than EGCs showing a positive Mlh1 expression [13/30 (43.3\%) vs. 21/79 (26.6\%), $\mathrm{P}=0.094$ ]. Adenomas with a loss of Mlh1 expression were found in only three tumors of elderly female patients (9.6\%) (data not shown). Loss of Mlh1 expression was closely associated with elevated macroscopic classification $(\mathrm{P}=0.04)$ and oncological family history $(\mathrm{P}=0.03)$. No significant difference was noted between the expression of Mlh1 and other clinicopathological or patient characteristics (Table III).

Among the elderly patients ( $\geq 65$ years), the females had significantly more EGCs with loss of Mlh1 expression than EGCs with a positive Mlh1 expression [13/22 (59.1\%) vs. 17/57 (29.8\%), P=0.016]. Furthermore, a family history of GC and an oncological family history were associated more often with EGCs with loss of Mlh1 expression than EGCs with a positive Mlh1 expression [13/22 (59.1\%) vs. $19 / 57$ (33.3\%), $\mathrm{P}=0.037$ and $17 / 22(77.3 \%)$ vs. $29 / 57$ (50.9\%), $\mathrm{P}=0.033$, respectively] (Table IV).

However, no correlation was found between P53 expression and phenotypic expression and any of the clinicopathological or patient data.

Relationship between Mlh1 expression and P53 and phenotypic expression in early gastric cancer. P53 overexpression 
Table III. Relationship between clinicopathological characteristics and Mlh1 expression in early gastric cancer.

\begin{tabular}{lccc}
\hline & \multicolumn{3}{c}{ Mlh1 expression } \\
\cline { 2 - 3 } & Positive (n=79) & Negative (n=30) \\
\hline Gender (M:F) & $58: 21$ & $17: 13$ & 0.094 \\
Age (mean \pm SD) & $69.7 \pm 9.1$ & $69.6 \pm 9.1$ & 0.903 \\
Elevated/flat, depressed & $38: 41$ & $21: 9$ & 0.040 \\
Histologic type (tub:pap:por/sig) & $64: 12: 3$ & $20: 9: 1$ & 0.216 \\
Depth of invasion (m:sm) & $76: 3$ & $26: 4$ & 0.070 \\
Regular alcohol intake (\%) & $38(48.1)$ & $13(43.3)$ & 0.660 \\
Current or ever smoker (\%) & $43(54.4)$ & $22(73.3)$ & 0.683 \\
Oncological family history (\%) & $39(49.4)$ & $16(53.3)$ & 0.030 \\
Family history of gastric cancer $(\%)$ & $26(32.9)$ & 0.051 \\
\hline
\end{tabular}

Tub, tubular adenocarcinoma; Pap, papillary adenocarcinoma; Por, poorly differentiated adenocarcinoma; Sig, signet ring cell adenocarcinoma.

Table IV. Relationship between Mlh1 expression and clinicopathological and patient characteristics in elderly ( $\geq 65$ years of age) patients with early gastric cancer.

\begin{tabular}{lccc}
\hline & \multicolumn{2}{c}{ Mlh1 expression } & P-value \\
\cline { 2 - 4 } & Positive $(\mathrm{n}=57)$ & Negative $(\mathrm{n}=22)$ \\
\hline Gender (M:F) & $40: 17$ & $9: 13$ & 0.016 \\
Age (mean \pm SD) & $74.4 \pm 5.4$ & $74.0 \pm 5.3$ & 0.903 \\
Elevated/flat, depressed & $33: 24$ & $17: 5$ & 0.109 \\
Oncological family history (\%) & $29(50.9)$ & $13(59.3)$ & 0.033 \\
Family history of gastric cancer $(\%)$ & $19(33.3)$ & 0.037 \\
\hline
\end{tabular}

occurred significantly more often in EGCs with a positive Mlh1 expression than in EGCs with loss of Mlh1 expression [28/79 $(35.4 \%)$ vs. $2 / 30$ (6.7\%), $\mathrm{P}=0.002]$. HGM expression was found significantly more often in GCs with loss of Mlh1 expression than in GCs with a positive Mlh1 expression [25/30 (83.3\%) vs. 46/79 (58.2\%), $\mathrm{P}=0.017$ ] (Table V).

Multivariate analysis of factors related to Mlh1 expression. Multivariate analysis using the logistic regression model showed that female gender $(\mathrm{P}=0.044 ; \mathrm{OR}=21.1 ; 95 \% \mathrm{CI} 1.08-408)$ and $\mathrm{P} 53$ expression $(\mathrm{P}=0.014 ; \mathrm{OR}=19.6 ; 95 \%$ CI 1.80-211) correlated with Mlh1 expression in elderly patients ( $\geq 65$ years of age) with EGC.

\section{Discussion}

The present study focused on endoscopically resected early gastric neoplasias and showed that loss of Mlh1 expression in EGCs in elderly individuals occurs more significantly in females than in males. In addition, our results indicate that the frequency of aberrant Mlh1 expression in EGCs increases significantly in patients with an oncological family history and elevated gross type.

Numerous GCs develop through defects in DNA MMR genes, such as $M L H 1$ or $M S H 2$, or tumor suppressor genes, such as P53 (12). Evidence of $M L H 1$ hypermethylation is
Table V. Relationship between Mlh1 expression and P53 and phenotypic expression in early gastric cancer.

\begin{tabular}{lccc}
\hline $\begin{array}{l}\text { Positive } \\
\text { expression }\end{array}$ & \multicolumn{2}{c}{ Mlh1 expression } & P-value \\
\cline { 2 - 3 } & Positive $(\mathrm{n}=79, \%)$ & \\
\hline P53 & $28(35.4)$ & $2(6.7)$ & 0.002 \\
HGM & $46(58.2)$ & $25(83.3)$ & 0.017 \\
CD10 & $25(31.6)$ & $5(16.7)$ & 0.120 \\
MUC2 & $14(17.7)$ & $16(53.3)$ & 0.550 \\
\hline
\end{tabular}

noted in approximately 20-30\% of differentiated carcinomas (11-13). Epigenetic methylation-associated inactivation of the MLH1 MMR gene is a potent trigger of MSI, especially high-frequency MSI (MSI-H) (17). Moreover, a strong correlation has been noted between MSI and methylation analysis, as well as MMR protein immunoexpression. Thus, immunohistochemistry is a rapid and cost-effective method for identifying MMR-gene alterations (31-34). As previously reported, MLH1 methylation is frequently observed in GCs of elderly patients $(18,19)$ and MSI-H GCs are identified by their expanding growth pattern (20). Similarly, in this study 
GC with loss of Mlh1 expression was associated with elevated gross type. In colorectal types of cancer, Breivik et al found that MSI occurred most frequently in young male and older female patients (35). In GC, the frequency of the promoter methylation of $M L H 1$ and the lack of Mlh1 expression increases with advancing age $(9,18,21)$. In our study, GCs with loss of Mlh1 expression were statistically significant in the elderly female patients. In GAs, loss of Mlh1 expression was detected in only three tumors of elderly female patients. While three studies have indicated a trend of association between female gender and immunohistochemical negativity for Mlh1 or for the MSI phenotype in GCs, the samples used in these studies were surgical tissues $(13,36,37)$. The results from these studies suggest differences between the genders in the molecular pathways of gastric neoplasia in elderly patients. Our study is the first to report on the relationship between gender and Mlh1 expression in early gastric neoplasia resected by endoscopy in elderly patients. Previous results from animal experiments have shown a protective effect of female gender on inflammation-associated cancer, which is attributed to estrogen (38). This mechanism may account for the frequent aberrant Mlh1 expression noted in older female patients with early gastric neoplasias. However, this mechanism has yet to be elucidated.

The association of MSI and family history of GC is controversial. In five studies, correlations between MSI and GC-positive family history were found (39-43). In particular, Shinmura et al noted an association between MSI and GC familial clustering in early GC, but not in advanced-stage GC (40). Kanemitsu et al indicated that MSI is induced by a deficiency in the MMR protein expression (42). Our analyses using endoscopically resected EGCs showed a significant association between aberrant Mlh1 expression and GC-positive family history in elderly patients. The association between MSI and family history may reflect exposure to environmental or dietary factors shared by close relatives. Taken together, these causal factors in familial GC may also be associated with MSI in the early stages of carcinogenesis.

On the basis of cellular phenotype, obvious differences occur in the biological behavior of gastric vs. intestinal phenotype of GCs. Gastric phenotype GCs are considered to have greater invasiveness and metastatic potential than intestinal phenotype GCs (28). Concerning the relationship between genetic/epigenetic alterations and phenotypes, DNA methylation of MLH1 occurred more frequently in GCs with gastric phenotype. Furthermore, Endo et al reported that MSI was detected more frequently in gastric phenotype GCs than in intestinal phenotype GCs (26). Findings of our previous and current studies showed that gastric phenotype EGCs were significantly associated with loss or reduction of Mlh1 expression (44). However, we did not find any correlation between phenotypic expression and other clinicopathological or patient data. Thus, our data and those of previous reports support the correlation between cellular phenotype and Mlh1 alterations. However, more studies are required to clarify the mechanisms of this association.

In the present study, P53 overexpression was found in $27.5 \%$ of EGCs and was inversely associated with loss of Mlh1 expression. This finding is consistent with previous reports $(12,45)$. However, the relationship between P53 expression and clinicopathological factors was not determined. It has been reported that $H$. pylori mediated the up-regulation of activationinduced cytidine deaminase results in the accumulation of nucleotide alterations in the P53 tumor suppressor gene in gastric cells in vitro (46). Thus, P53 overexpression may be directly affected by $H$. pylori infection and not by individual characteristics.

In conclusion, this study showed that EGC with Mlh1 silencing exhibits characteristics, such as age, gender, family history and tumor growth pattern. These data should be used for the screening and surveillance of GC, particularly in elderly individuals. Further investigations into the relationship between MMR methylation and patient and tumor characteristics through in vitro and/or in vivo studies are required to thoroughly elucidate the molecular interactions involved.

\section{References}

1. Parkin DM: International variation. Oncogene 23: 6329-6340, 2004.

2. Parkin DM, Brey F, Ferlay J, et al: Global cancer statistics, 2002. CA Cancer J Clin 55: 74-108, 2005.

3. Arai T, Esaki Y, Inoshita N, et al: Pathologic characteristics of gastric cancer in the elderly: a retrospective study of 994 surgical patients. Gastric Cancer 7: 154-159, 2004.

4. Eslick GD: Helicobacter pylori infection causes gastric cancer? A review of the epidemiological, meta-analytic, and experimental evidence. World J Gastroenterol 21: 2991-2999, 2006.

5. Berinini M, Barbi S, Roviello F, et al: Family history of gastric cancer: a correlation between epidemiologic findings and clinical data. Gastric Cancer 9: 9-13, 2006.

6. Kawasaki K, Kanemitsu K, Yasuda T, et al: Family history of cancer in Japanese gastric cancer patients. Gastric Cancer 10: 173-175, 2007.

7. World Cancer Research Fund, American Institute for Cancer Research, Food, Nutrition, Physical Activity and Prevention of Cancer: A Global Perspective. AICR, 2007.

8. Jemal A, Clegg LX, Ward E, et al: Annual report to the nation on the status of cancer, 1975-2001, with a special feature regarding survival. Cancer 101: 3-27, 2004.

9. Arai T and Takubo K: Clinicopathologicalal and molecular characteristics of gastric and colorectal carcinomas in the elderly. Pathol Int 57: 303-314, 2007.

10. Yashima K, Sasaki S, Koda M, Kawaguchi K, Harada K and Murawaki Y: Premalignant lesions in gastric cancer. Clin $\mathrm{J}$ Gastroenterol 3: 6-12, 2010.

11. Koseki K, Takizawa T, Koike M, Ito M, Nihei Z and Sugihara K: Distinction of differentiated type early gastric carcinoma with gastric type mucin expression. Cancer 89: 724-732, 2000.

12. Tamura G: Alteration of tumor suppressor and tumor-related genes in the development and progression of gastric cancer. World J Gastroenterol 14: 192-198, 2006.

13. Hong SH, Kim HG, Chung WB, et al: DNA hypermethylation of tumor-related genes in gastric carcinoma. J Korean Med Sci 20: 236-241, 2005.

14. Maesawa C, Tamura G, Suzuki Y, Ogasawara, Sakata K, Kashiwaba $\mathrm{M}$ and Satodate R: The sequential accumulation of genetic alterations characteristic of the colorectal adenomacarcinoma sequence does not occur between gastric adenoma and adenocarcinoma. J Pathol 176: 249-258, 1995.

15. Yamazaki K, Tajima Y, Makino R, et al: Tumor differentiation phenotype in gastric differentiated-type tumors and its relation to tumor invasion and genetic alterations. World J Gastrenterol 28: 3803-3809, 2006.

16. Fleisher AS, Esteller M, Wang S, et al: Hypermethylation of the hMLH1 gene promoter in human gastric cancers with microsatellite instability. Cancer Res 59: 1090-1095, 1999.

17. Fleisher AS, Esteller M, Tamura G, et al: Hypermethylation of the hMLH1 gene promoter is associated with microsatellite instability in early human gastric neoplasia. Oncogene 20: 329-335, 2001. 
18. Nakajima T, Akiyama Y, Shiraishi J, et al: Age-related hypermethylation of the hMLH1 promoter in gastric cancers. Int J Cancer 94: 208-211, 2001.

19. Kim HG, Lee S, Kim DY, et al: Aberrant methylation of DNA mismatch repair genes in elderly patients with sporadic gastric carcinoma: a comparison with younger patients. J Surg Oncol 101: 28-35, 2010.

20. Ceccarelli M, Sera F, Costantini RM, Nesi G, Palli D and Ottini L: Gastric cancer with high-level microsatellite instability: target gene mutations, clinicopathologic features and long-term survival. Hum Path 39: 925-932, 2008.

21. Baylin SB and Herman JG: DNA hypermethylation in tumorigenesis: epigenetics joins genetics. Trends Genet 16: 168-174, 2000.

22. Egashira Y, Shimoda T and Ikegami M: Mucin histochemical analysis of minute gastric differentiated adenocarcinoma. Pathol Int 49: 55-61, 1999.

23. Yoshino T, Shimoda T, Saito A, Nakanishi Y, Tajima Y, Shirasu T and Miura S: Macroscopic features of differentiated adenocarcinoma with gastric or intestinal phenotype expression in early gastric cancer. Stomach Intestine 34: 513-525, 1999.

24. Koseki K, Takizawa T, Koike M, Ito M, Nihei Z and Sugihara K: Distinction of differentiated type early gastric carcinoma with gastric type mucin expression. Cancer 89: 724-732, 2000.

25. Koseki K, Takizawa T, Koike M, et al: Subclassification of welldifferentiated gastric cancer with reference to biological behavior and malignancy, gastric type vs. intestinal type, and papillary carcinoma vs. tubular carcinoma. Stomach Intestine 34: 507-512, 1999.

26. Endoh Y, Tamura G, Sakata K, et al: Genetic analysis of differentiated-type adenocarcinomas of the stomach with gastric phenotype and intestinal phenotype. Stomach Intestine 34 539-544, 1999.

27. Tajima Y, Shimoda T, Nakanishi Y, et al: Gastric and intestinal phenotypic marker expression in gastric carcinomas and its prognostic significance: immunohistochemical analysis of 136 lesions. Oncology 61: 212-220, 2001.

28. Kabashima A, Yao T, Sugimachi K and Tsuneyoshi $M$ Relationship between biologic behavior and phenotypic expression in intramucosal gastric carcinoma. Hum Pathol 33: 80-86, 2002.

29. Japanese Gastric Cancer Association: Japanese classification of gastric carcinoma - 2nd English edition. Gastric Cancer 1: 10-24, 1998.

30. Schlemper RJ, Riddell RH, Kato Y, et al: The Vienna classification of gastrointestinal epithelial neoplasia. Gut 47: 251-255, 2000.

31. Dietmaier W, Wallinger S, Bocker T, Kullmann F, Fishel R and Ruschoff J: Diagnostic microsatellite instability: definition and correlation with mismatch repair protein expression. Cancer Res 57: 4749-4756, 1997

32. Mizoshita T, Tsukamoto T, Cao X, et al: Microsatellite instability is linked to loss of hMLH1 expression in advanced gastric cancers: lack of a relationship with the histological type and phenotype. Gastric Cancer 8: 164-172, 2005.
33. Thibodeau SN, French AJ, Cunningham JM, et al: Microsatellite instability in colorectal cancer: different mutator phenotypes and the principal involvement of hMLH1. Cancer Res 58: 1713-1718, 1998.

34. Chaves P, Cruz C, Lage P, Claro I, Cravo M, Leitao CN and Soares J: Immunohistochemical detection of mismatch repair gene proteins as a useful tool for the identification of colorectal carcinoma with the mutator phenotype. J Pathol 191: 355-360, 2000.

35. Breivik J, Lothe RA, Meling GI, et al: Different genetic pathways to proximal and distal colorectal cancer influenced by sex-related factors. Int J Cancer 74: 664-669, 1997.

36. French AJ, Petroni G, Thibideau SN, et al: Allelic imbalance of $8 p$ indicates poor survival in gastric cancer. J Mol Diagn 6: 243-253, 2004

37. Beghelli S, Manzoni G, Barbi S, et al: Microsatellite instability in gastric cancer is associated with better prognosis in only stage II cancers. Surgery 139: 347-356, 2006.

38. Schwartz AG, Whitcomb JM, Nyce JW, Lewbart ML and Pashko LL: Dehydroepiandrosterone and structural analogs: a new class of cancer chemopreventive agents. Adv Cancer Res 51: 391-424, 1988.

39. Keller G, Rotter M, Vogelsang H, et al: Microsatellite instability in adenocarcinomas of the upper gastrointestinal tract. relation to clinicopathologicalal data and family history. Am J Pathol 147: 593-600, 1995.

40. Shinmura K, Yin W, Isogaki J, et al: Stage-dependent evaluation of microsatellite instability in gastric carcinoma with familial clustering. Cancer Epidemiol Biomarkers Prev 6: 693-697, 1997.

41. Ottini L, Palli D, Falchetti M, et al: Microsatellite instability in gastric cancer is associated with tumor location and family history in a high-risk population from Tuscany. Cancer Res 57: 4523-4529, 1997.

42. Kanemitsu K, Kawasaki K, Nakamura M, et al: MSI is frequently recognized among gastric cancer patients with a family history of cancer. Hepatogastroenterology 54: 2410-2414, 2007.

43. Pedrazzani C, Corso G, Velho S, et al: Evidence of tumor microsatellite instability in gastric cancer with familial aggregation. Familial Cancer 8: 215-220, 2009.

44. Hara A, Yashima K, Yasugi A, et al: Expression of Fhit, Mlh1, p $16^{\mathrm{INK} 4 \mathrm{~A}}$ and E-cadherin in early gastric neoplasia: Correlation with histological grade and gastric phenotype. Oncol Rep 18: 553-559, 2007.

45. Martinez R, Schackert HK, Plaschke J, Baretton G, Appelt H and Schackert G: Molecular mechanisms associated with chromosomal and microsatellite instability in sporadic glioblastoma multiforme. Oncology 66: 395-403, 2004.

46. Matsumoto $\mathrm{Y}$, Marusawa $\mathrm{H}$, Kinoshita $\mathrm{K}$, et al: Helicobacter pylori infection triggers aberrant expression of activationinduced cytidine deaminase in gastric epithelium. Nat Med 13: 470-476, 2007. 